\title{
Rincón científico
}

\section{COMUNICACIONES}

2Pilar Domínguez Oliván

'Profesora TUE de Enfermería Geriátrica, Escuela Universitaria de Ciencias de la Salud de Zaragoza. Máster en Gerontología Social.

${ }^{2}$ Profesora TEU de Teoría y Técnica Fisioterápica Especial II, Escuela Universitaria de Ciencias de la Salud de Zaragoza.

Correspondencia:

Ma Dolores Azúa Blanco

Escuela Universitaria de Ciencias de la Salud de Zaragoza

C/ Domingo Miral s/n. 50009-Zaragoza

Tfno.: 976761751 (Ext. 4458)

Fax: 976761752

E-mail: neka@unizar.es
Complicaciones personales de incontinencia fecal

\author{
Personal complications in patients \\ with fecal incontinence syndrome
}

\section{RESUMEN}

La pérdida involuntaria de masa fecal, no siendo realmente una patología en sí misma, es a veces, para la persona que la padece, más importante que la situación que la provoca. Róbale el cuerpo y úsalo, es más, róbale también su mente, sus ideas, sus sensaciones, sentimientos y miedos: ¿notas su tensión psíquica al cerrar la puerta de casa? ¿Lloras de rabia y de tristeza al cambiar la ropa manchada? Esto es sólo una milésima parte de lo que ocurre. Imagínate con 70 u 80 años, con un cuerpo cansado y lento, con la mente no muy dispuesta a luchar y con este "problemilla".

\section{PALABRAS CLAVE}

Incontinencia fecal, repercusión física, repercusión psíquica, repercusión social, repercusión económica.

\section{SUMMARY}

The involuntary loss of fecal mass, not being really a disease in itself is, at times, to the person who suffers, more important that the situation that provokes. Steal the body and use it, it is more, steal their mind, their thoughts, their sensations, feelings and fears; Do you feel his mental tension when closing the door? Do you cry of anger and sadness when changing stained clothes? This is just a thousandth part of what happens. Imagine at age of 70 or 80 , with a tired and slow body, with the mind not very willing to fight and with this "little problem".

\section{KEY WORDS}

Fecal incontinence, physical impact, psychological impact, social impact, economical impact.

\section{INTRODUCCIÓN}

La incontinencia fecal, al igual que la tos, el dolor, la fiebre, etc., no es una patología en sí misma. Es un síndrome o una manifestación clínica de una enfermedad. Esto, tan obvio para los sanitarios, no pasa de ser un mero tecnicismo para el anciano o la anciana, ya que las repercusiones personales de dicha situación pueden llegar a tener una gravedad elevada.

Con esta revisión no pretendemos hallar soluciones a las diversas situaciones que se producen, ni siquiera pretendemos buscarlas; nos limitamos a exponer las tremendas repercusiones a las que conduce la incontinencia fecal. Tampoco tratamos de explicar las causas que pueden desembocar en esta temida situación, no es el objetivo de esta revisión.

No obstante a la expuesto, sí tenemos que expresar la necesidad de que, ante esta situación, es indiscutible la necesidad de realizar una buena valoración integral. La valoración clínica es precisa, no sólo para la búsqueda de las causas, sino también para investigar las repercusiones en la salud física que la incontinencia fecal puede ejercer sobre la persona. La valoración de la función física es también necesaria, principalmente en lo referente al grado de dependencia en lo relativo a su cuidado (higiene, nutrición, etc.); no podemos obviar la necesidad de una buena valoración psíquica o mental, principalmente en el área afectiva, para poder contestar a la pregunta " ¿Hay repercusión psicoafectiva en este persona?”; y, por último y en relación con la valoración social, la tan importante repercusión en todas las actividades sociales, desde la propia familia hasta la macrosociedad en la que está inmerso el anciano/a. No olvidemos que la problemática presentada en cualquiera de estas "esferas" repercute indefectiblemente sobre el resto, comprometiendo y complicando los diagnósticos, médicos y de enfermería, y la asistencia del anciano o anciana con incontinencia fecal en cualquiera de sus formas. 
191 La incontinencia fecal es una pérdida involuntaria de heces, pudiendo producirse desde una ligera filtración ocasional de heces con el paso de gases, hasta una pérdida completa del control de las deposiciones, independientemente de la frecuencia o intensidad (1), en momentos inapropiados (2). Según estudios realizados por el Dr. Fermín Mearin, del 3\% al 7\% de la población sufre incontinencia fecal, principalmente las mujeres, las personas mayores de 65 años y las personas con dificultad en la movilización. Es uno de los síndromes geriátricos que mayor repercusión tienen en la calidad de vida del anciano y de su cuidador. Es tristemente una gran desconocida en el ámbito sanitario, a pesar de ser la causa de que muchos pacientes dejen de vivir en sus domicilios. La asociación de incontinencia fecal con la incontinencia urinaria (doble incontinencia o mixta) se observa frecuentemente en las personas mayores que comienzan con una incontinencia fecal.

La situación de la incontinencia fecal suele ser un problema oculto por diversas razones, miedo, vergüenza, desconcierto, falsas creencias, aceptación... principalmente, se basa en el desconocimiento de la situación. Todo ello implica que, con frecuencia, el paciente no refiera su existencia, debiéndose preguntar específicamente por esta cuestión y observar la reacción del anciano/a.

La importancia de la incontinencia fecal no radica tan sólo en ser una de las principales causas de institucionalización del anciano, también debe su importancia al gasto económico que suponen para la sociedad los tratamientos, tanto curativos como paliativos, así como la carga familiar que supone esta situación y su atención; adquiere mayor importancia cuando se analizan las repercusiones en la persona que padece ente síndrome, repercusiones personales que son tremendamente importantes. Comenzando por la situación emocional que supone para mu-

\begin{tabular}{|ll|}
\hline \multicolumn{2}{|c|}{ Tabla 1. Repercusiones personales } \\
\hline $\begin{array}{l}\text { - En la salud física } \\
\text { - En la salud psíquica }\end{array}$ & - En el bienestar social \\
\hline
\end{tabular}

chos pacientes geriátricos, ya que constituye una regresión humillante en la función corporal, que origina un grave deterioro de la actividad y la relación social.

Las repercusiones a nivel personal son variadas y, como hemos dicho con anterioridad, todas ellas se acaban relacionando y comprometiendo la estabilidad de la personas. Básicamente las podemos encuadrar en cuatro grandes áreas de repercusión (Tabla 1): 1) a nivel de salud física, 2) en relación al bienestar psíquico; 3) referidas al bienestar social y, por último, no menos importante, 4) la repercusión económica para el propio paciente.

\section{Salud física (Tabla 2)}

Se produce una situación de riesgo de deterioro de la integridad cutánea por varias causas, teniendo presente, en principio, que el órgano cutáneo de las personas mayores presenta modificaciones inherentes a la edad avanzada y que predisponen a la aparición de problemas de salud cutánea por pequeñas causas, a lo que hay que sumar el problema de la incontinencia fecal, lo que implica la acción de sustancias químicas y de otros tipos procedentes de las sustancias de deshecho, siendo estas últimas favorecedoras directas de infecciones, mientras que las sustancias químicas provocan irritación con la consiguiente fricción y rascamiento ocasionado por el picor producido, lo que rápidamente ocasionará pequeños traumatismos y excoriaciones con alta posibilidad de infecciones. Los dispositivos paliativos al uso y la presión provocada por éstos sobre la piel comprometen y alteran la integridad cutánea, siendo

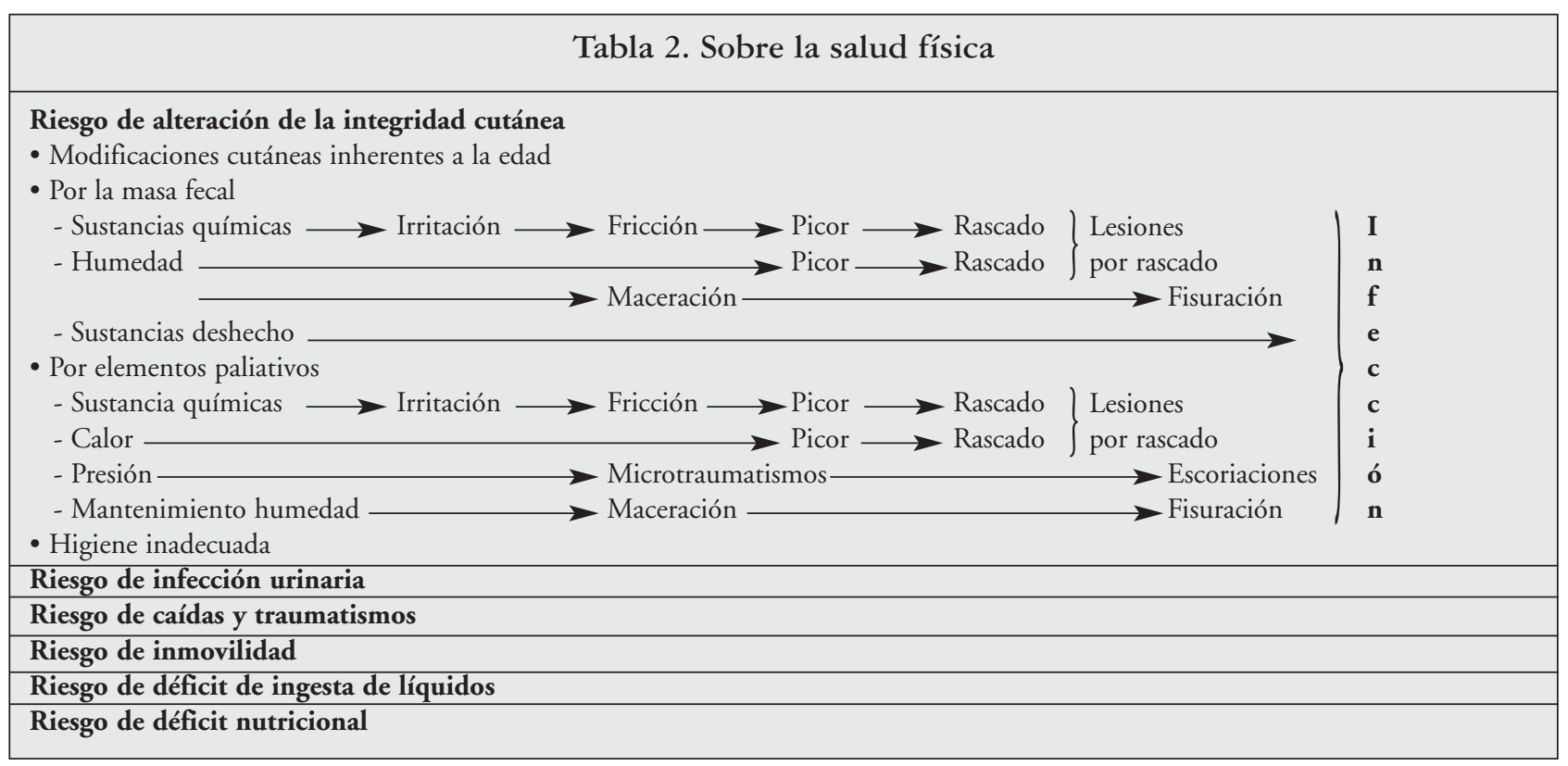


causa posible de excoriaciones que dejan la puerta abierta a infecciones.

La humedad constante que la sustancia líquida de la incontinencia fecal provoca en la piel favorece no sólo la aparición de prurito, también es causa de la maceración cutánea, siendo ésta la provocadora de fragilidad cutánea y fácil fisuración de la zona, siendo altamente probable la aparición de infecciones per se favorecidas, en mayor medida, por la materia expulsada involuntariamente del cuerpo; pero la humedad, como decíamos anteriormente, es también causante de picor, provocando -junto con la irritación y otros factores- que la persona se rasque la zona afectada, lo que acaba ocasionando todas las lesiones secundarias al rascado (liquenificación, escoriación, infección, etc.) y más picor secundario a estas lesiones. Esta humedad cutánea se ve favorecida también por el contacto en la piel y las mucosas de algunos dispositivos (absorbente y bolsas perineales).

Los dispositivos paliativos al uso y la presión provocada sobre la piel comprometen y alteran la integridad cutánea, siendo causa posible de excoriaciones que dejan la puerta abierta a infecciones.

El calor producido por los dispositivos absorbentes, principalmente, junto con las sustancias utilizadas (desodorantes, colonias, etc.), provocan, mediante diversas vías de acción, picor con su inevitable rascado y posteriores lesiones secundarias.

La higiene inadecuada, haciendo referencia tanto al déficit de la misma, ocasiona problemas de fácil comprensión; tantas como el exceso de higiene, que conducirá a una eliminación del manto ácido cutáneo lipídico, viéndose afectadas todas las funciones que dicho manto ejerce en la piel, diminuidas o incluso eliminadas, repercutiendo de esta manera en el mantenimiento de una piel en perfectas condiciones, suave, sin fisuras, con capacidad de protección frente a diversos microorganismos, etc.

$\mathrm{Al}$ describir el riesgo de deterioro de la integridad cutánea no podemos olvidar el riesgo de escaras e, incluso, el riesgo de aparición de úlceras por presión, debido en parte a todo lo anteriormente expuesto y al hecho de que el uso de dispositivos absorbentes, entre otros elementos, favorece el roce y los pequeños traumatismos (3), circunstancias que se agravan con el sedentarismo, cada vez más pertinaz, de nuestros mayores, pero aumentado por la incomodidad de los dispositivos, miedo a..., es decir, por las repercusiones psíquicas.

Otras complicaciones o repercusiones que observamos en estas situaciones son: el riesgo de infección urinaria por el mal uso o simplemente uso de dispositivos tales como sondas rectales, bolsas anales, tapones anales o bien simplemente absorbentes.

El riesgo de caídas y traumatismos como consecuencia del deslizamiento sobre suelo resbaladizo mojado/sucio por escapes de materia fecal líquida o semilíquida como consecuencia de la mala colocación del dispositivo en uso, u otras causas de orden diverso.
La situación de riesgo de inmovilidad progresiva, como consecuencia del miedo a padecer caídas (experiencias negativas), o bien simplemente por la incomodidad que causan los elementos paliativos, o bien miedo o más bien vergüenza de que se note el dispositivo.

El riesgo de déficit de la ingesta de líquidos, ya que se produce una reducción voluntaria en la ingesta de líquidos de forma alarmante, con el fin de disminuir la frecuencia de las pérdidas, junto con un intento, en ocasiones, de hacer más dura la masa fecal para evitar en lo posible los escapes o, al menos, que estos no sean tan líquidos. La disminución de la ingestión de líquidos puede, incluso, llegar a causar otros problemas a nivel renal, digestivo...

Igualmente, el riesgo de déficit nutricional es fácil de observar, básicamente por las mismas razones que la disminución en la ingesta de líquidos y ante la idea de "si no ingiero, no hay masa fecal, no hay pérdida”.

La persona mayor que padece este síndrome experimenta una serie de sensaciones muy diversas. Unas son lo que podríamos denominar experiencias físicas, tales como el olor, la tensión muscular, la imaginación..., otras veces son ideas mentales de sí mismo ante el pensamiento de diversas situaciones (Tabla 3). Todas estas experiencias e ideas ejercen un influencia negativa sobre el bienestar psíquico e, igualmente, traen consecuencias negativas sobre los aspectos de bienestar social.

\section{Bienestar psiquíco}

Encontraremos diversas actitudes en la persona que reflejarán la influencia psíquica de la incontinencia sobre su bienestar psicológico (Tabla 4).

Surgen sentimientos de culpa, vergüenza e, incluso, negación de la evidencia clara de incontinencia (pudiendo dar largas explicaciones para justificar olores y manchas, incluso esconder la ropa sucia), dificultando la búsqueda de ayuda, improvisándose medios poco adecuados y frustrantes (4):

\section{Tabla 3. Sensaciones/pensamientos/ideas}

- Estar de pie en un charco sucio con los "amigos" riéndose de él.

- Tener que explicar a mis hijos/nietos que me ensucio y que ensucio la cama como un crío.

- No tener lavabo a mano.

- Encontrar un lavabo pero con una larga cola.

- Ir al cine pero dejar rápidamente el asiento para ir al lavabo.

- Ver alejarse a la gente del autobús de mi lado debido al olor.

- He de estar cerca del lavabo a todas horas o me pasará algo.

- Debo de oler muy mal y estar muy sucio.

- Tengo miedo a salir en público.

- Me odio y carezco de confianza.

- Soy feo.

- Si no puedo llegar a controlar esta parte de mi cuerpo, ¿cuál será la siguiente?

- No valgo para nada y no tengo ninguna esperanza. 
193

\begin{tabular}{|c|c|}
\hline \multicolumn{2}{|c|}{ Tabla 4. Bienestar psíquico } \\
\hline $\begin{array}{l}\text { - Alteración de la autoestima } \\
\text { - Alteración de la imagen } \\
\text { corporal } \\
\text { - Ansiedad, preocupación } \\
\text { - Combate inefectivo }\end{array}$ & $\begin{array}{l}\text { - Vergüenza } \\
\text { - Aislamiento psíquico } \\
\text { - Irritabilidad } \\
\text { - Otros... }\end{array}$ \\
\hline
\end{tabular}

- Trastorno e, incluso, pérdida de la autoestima, basado en la percepción negativa de uno mismo, expresada en sentimientos de vergüenza y negación a realizar las tareas o actividades (nuevas o rutinarias).

- Trastornos de la imagen corporal, manifestados por sentimientos negativos sobre su cuerpo o temor al rechazo.

- Ansiedad y preocupación como respuesta a sentir incertidumbre y desamparo, expresadas a través de cambios de conducta. Esta ansiedad es la traducción, junto con otras actitudes, del miedo a sufrir reprimendas o rechazo de las personas de su entorno.

- Enfrentamiento individual inefectivo, debido a una incapacidad de pedir ayuda o para hacer frente a la situación.

Otras actitudes se pueden observar como consecuencia de todo lo expuesto:

- Vergüenza.

- Aislamiento psíquico.

- Depresión.

- Dependencia.

- Irritabilidad.

- Ira.

- Tensión

- Frustración.

- Vulnerabilidad.

\section{Consecuencias sociales}

Los problemas de salud derivados de la enfermedad y las complicaciones surgidas por la incontinencia, como las repercusiones psíquicas de este síndrome, conducen a repercusiones negativas en el amplio abanico de actividades sociales, tanto a nivel familiar como en el ámbito de las amistades, etc., apareciendo en diferente grado, según el tipo y la gravedad de la incontinencia y de la propia personalidad del anciano. Dicho de otro modo, son numerosos los pacientes que adoptan hábitos sociales y conductas alejadas de las relaciones personales por esta causa, dando lugar a (Tabla 5):

- Deterioro de la interacción social y aislamiento, manifestado por la búsqueda de soledad, tristeza, sentimiento de soledad, inseguridad en público. Todo ello contribuye a:

- Ausencia de relaciones personales satisfactorias.

- Pérdida e incapacidad para establecer relaciones interpersonales.

\begin{tabular}{|ll|}
\hline \multicolumn{2}{|c|}{ Tabla 5. Consecuencias sociales } \\
\hline - Deterioro-interacción & • Predisposición \\
social: aislamiento & a la institucionalización \\
- Alteración de desempeño & - Dejadez del aspecto \\
del rol & personal \\
- Disminución del interés & - Otras... \\
social & \\
- Alteración de la convivencia & \\
familiar/amistades & \\
\hline
\end{tabular}

- Aislamiento físico.

- Evidente riesgo de soledad

- Alteración del desempeño del rol o, lo que es lo mismo, la percepción de no tener uno real o un sentido en su vida (“A partir de ahora ¿qué vendrá?”: vergüenza ante el cónyuge y los hijos).

- Disminución por el interés en actividades recreativas o de ocio, debido a posibles pérdidas.

- Alteraciones importantes en el sistema familiar, tanto por parte de la inadaptación de los hijos, nietos... como del propio paciente.

En resumen, nos encontraremos problemas en estas actividades :

- Estrés en la familia, los amigos y los cuidadores.

- Abandono de las actividades domésticas, sociales y laborales, lo que conduce al aislamiento social y, poco a poco, a dejadez en el aspecto personal (pierde importancia).

- Predisposición a la institucionalización.

- Aislamiento social.

- Mayor necesidad de apoyo familiar y social.

- Dejadez en el aspecto personal.

\section{Repercusión económica}

Los costes económicos para la propia persona como, en su caso, para la familia, por no hablar para la sociedad, pese a no ser la más importante de las repercusiones, no cabe duda de que es un punto tremendamente importante, ya que no se trata solamente del precio de los dispositivos, bien sean los diferentes tipos de absorbentes, los conductores..., del aumento de la cantidad de ropa debido a la necesidad de mayor número de cambios de la misma, tanto de cama como de vestir, del gasto de lavandería (lavadora, plancha, jabón... tiempo), del trabajo que se ocasiona... Hay que tener presente que las propias complicaciones, principalmente físicas, también ocasionan gastos y a veces muy importantes (Tabla 6).

- Dispositivos.

- Ropa de cama y de vestir.

- Lavandería.

- Trabajo por parte del personal.

- Tratamiento de las complicaciones. 


\section{Tabla 6. Repercusiones económicas personales}

- Dispositivos

- Ropa de cama y de vestir

- Lavandería

- Trabajo por parte del personal

- Tratamiento de las complicaciones

Todo esto es causa de una influencia negativa sobre el bienestar psíquico e, igualmente, trae consecuencias negativas sobre los aspectos sociales. Pero hay que pensar que la alteración de la salud física también repercutirá en la psique y en la actividad social, así como los problemas a nivel psíquico repercuten en la actividad social y en la salud física, y los problemas sociales sobre las otras esferas.

- Valoración física, con el fin de valorar el grado o la existencia de discapacidades físicas que favorezcan la situación (AVD...).

- Valoración psíquica, valoración de la capacidad cognitiva, existencia de patologías psíquicas, etc., que impidan el reconocimiento de la necesidad de defecación. La valoración afectiva nos indicará si existe depresión, ansiedad, "respuesta sucia", etc.

- Valoración social, principalmente si existen discapacidades tanto físicas como psíquicas, ya que el respaldo social puede ser muy interesante como factor favorecedor de la situación.

\section{BIBLIOGRAFÍA}

1. Pérez del Molino J, Valencia Isarch T. Incontinencia urinaria. En: Síndromes y Cuidados en el Paciente Geriátrico, Guillén Llera F, Pérez del Molino J. Barcelona: Masson, 1994. p. 191-2.

2. The Merck Manual of Geriatrics. Barcelona: Doyma, 1992, p. 525-6 y 548-50.
3. Lago González Mo de la L. Valoración de riesgo de úlceras por presión en una Unidad de geriatría. Gerokomos 2007; 18 (3): 135-9.

4. Ruipérez Cantera I, Llorente Domingo P et al. Guía para auxiliares y cuidadores del anciano. Madrid: McGraw-Hill-Interamericana, 2003. p. 107-8. 